\title{
The computation of the pitch damping stability derivatives of supersonic blunt cones using unsteady sensitivity equations
}

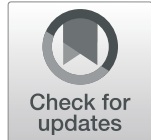

\section{Chenxi Guo and Yu-xin Ren * (1)}

\author{
* Correspondence: ryx@tsinghua. \\ edu.cn \\ Department of Engineering \\ Mechanics, Tsinghua University, \\ Beijing 100084, China
}

\begin{abstract}
The numerical methods for computing the stability derivatives of the aircraft by solving unsteady sensitivity equations which was proposed in our previous papers was extended to solve three-dimensional problems in this paper. Both the static and dynamic derivatives of the hypersonic blunt cone undergoing pitching oscillation around a fixed point were computed using the new methods. The predicted static derivative and dynamic derivative were found to be in reasonable agreement with the experimental data. For the present method, it is possible to distinguish the components of dynamic derivatives caused by different state parameters. It is found that $C_{m_{m}}$ and $C_{m_{q}}$ are usually of opposite signs and tend to eliminate each other, which makes $C_{m_{\dot{a}}}+C_{m_{q}}$ being much smaller than its individual components.

Another feature of this method is that the moment of pressure derivatives proposed in the present paper can be used to predict the contribution of each part of the blunt cone to the overall stability quantitatively. It is found that the head region is crucial for the static stability and the body region contributes most to the dynamic stability.

Keywords: Stability derivatives, Unsteady sensitivity equations, Three dimensional flows, Blunt cones, Numerical simulation
\end{abstract}

\section{Introduction}

The determination of the stability characteristics of atmospheric flight vehicles is the basis of the control system design, which is one of the most essential yet challenging stages in the whole process of aircraft development. A poor understanding or prediction of the stability characteristics may lead to a rise in costs and detrimental effects on the performance of the aircraft $[1,2]$. Therefore, it is necessary for designers to have proper knowledge of the stability characteristics, of which the stability derivatives are the key parameters.

The concept of stability derivatives was introduced by Byran [3] based on the assumption of linear relations between the aerodynamic forces/moments and instantaneous values of the disturbances of the kinematic variables. This model was very successful for flight at small angles of attack and with small disturbances. However, under extreme flight conditions involving high angle of attack, high pitch rate and/or gust responses, it

(c) The Author(s). 2019 Open Access This article is distributed under the terms of the Creative Commons Attribution 4.0 International License (http://creativecommons.org/licenses/by/4.0/), which permits unrestricted use, distribution, and reproduction in any medium, provided you give appropriate credit to the original author(s) and the source, provide a link to the Creative Commons license, and indicate if changes were made. 
is important to develop the aerodynamic models accounting for the nonlinear and unsteady effects. Some extended aerodynamic models can be found in [4-10] and others.

No matter which aerodynamic model is adopted, accurate evaluations of the aerodynamic response are essential [11], which can be carried out by wind tunnel experiments, flight tests, and computational fluid dynamics (CFD) simulations. The CFD approaches have great potentials in simulating wide ranges of flight conditions, predicting complete sets of data for the stability characteristics analysis and removing the interference effects of the model support [12], and have been developed considerably in recent years.

The CFD methods for computing the stability derivatives can be roughly divided into two categories. The first one is computing the flow fields first and then evaluating the stability derivatives using a certain parameter identification technique. These methods are very close to the experimental methods for evaluating the stability derivatives, and the only difference is that the aerodynamic forces and moments are computed instead of measured. One example of this type is the forced-oscillation approach, in which the stability derivatives can be computed by the integration of the periodic solutions of force/moment coefficients [13]. Ronch et.al [14-16]. used this approach to systematically study the stability derivatives of several models of aircraft in terms of various CFD solvers, such as RANS, harmonic balance, and linear frequency methods. There are several limitations to this approach. Firstly, only combinations of the stability derivatives such as $C_{m_{\alpha}}-k^{2} C_{m_{\dot{q}}}$ and $C_{m_{\dot{\alpha}}}+C_{m_{q}}$ can be computed. To separate $C_{m_{\dot{\alpha}}}$ and $C_{m_{q}}$, additional plunging motion must be considered besides the pitching motion. This approach is however not rigorous and will introduce additional errors. Secondly, the stability derivatives are assumed to be constant and their dependence on the reduced frequency is not known. More general approaches in terms of parameter identification techniques have been studied in $[17,18]$. Nevertheless, these approaches all require pre-assumed aerodynamic models.

The second category of CFD methods is directly computing the stability derivatives by solving the flow as well as the static sensitivity equations. This category of sensitivity equation based method was put forward by Godfrey and Cliff [19]. Limache [20] followed this approach and computed the pitch-rate derivatives of the airfoil under the steady motion. These methods are capable of computing various stability derivatives directly without relying on the parameter identification technique. However, the application of these sensitivity-based methods is confined to the study on static stability derivatives, since the basis of these methods is the static sensitivity equations. Similar methods based on automatic differentiation adjoint approach can be found in [3].

Ren [21] developed a sensitivity equation based method for computing the stability derivatives that account for the unsteady effects. This method is based on an extension of the conventional stability derivative model. Taking the relation between the pitching moment $C_{m}(t)$ and motion time history of the angle of attack $\alpha(t)$ as an example, he demonstrated that if $\alpha(t)$ can be expressed as a convergent Taylor series and the pitching moment is the function of $\alpha(t)$ and its time derivatives of various order

$$
C_{m}(t)=C_{m}(\alpha(t), \dot{\alpha}(t), \ddot{\alpha}(t), \cdots),
$$

the unsteady sensitivity equations can be derived. Then the stability derivatives can be 
computed directly from the solution of the sensitivity equations. This method does not rely on the linear or linearized aerodynamic model and takes the unsteady effects into consideration. Furthermore, this method is capable of predicting all stability derivatives from a single maneuver because of the use of information obtained from the sensitivity equations. In [22], this method was extended to compute the stability derivatives associated with supersonic flow with shock waves. The behavior of the solution of the sensitivity equations in the vicinity of shock waves was analyzed. In these papers, only simple two-dimensional cases were studied. Further studies are needed to validate the proposed method using more realistic three-dimensional test cases.

In this paper, the longitudinal stability derivatives of a blunt cone in supersonic flows are studied by solving the three-dimensional unsteady sensitivity equations. The results are compared with the experimental data to demonstrate the validity of this method. Besides further validation of the unsteady sensitivity equation based method for computing the stability derivatives, the main purpose of the present paper is to analyze the behaviors of $C_{m_{\dot{\alpha}}}$ and $C_{m_{q}}$ which, instead of the combination $C_{m_{\dot{\alpha}}}+C_{m_{q}}$, can be computed individually using the present method. Based on the distributions of sensitivity variables solved by the sensitivity equations, the contributions to the overall stability of any part of the cone can be evaluated quantitatively.

\section{Sensitivity equations and the numerical approaches}

In the non-inertial frame of reference fixed on the aircraft, the three-dimensional Euler equations in conservation form are show in Eq.(1) as follows

$$
\frac{\partial \mathbf{U}}{\partial t}+\frac{\partial \mathbf{F}}{\partial x}+\frac{\partial \mathbf{G}}{\partial y}+\frac{\partial \mathbf{H}}{\partial z}=\mathbf{R}
$$

where $\mathbf{U}$ is the vector of conservation variables and $\mathbf{F}$, and $\mathbf{G}$ and $\mathbf{H}$ are the inviscid fluxes:

$$
\mathbf{U}=\left[\begin{array}{c}
\rho \\
\rho u \\
\rho v \\
\rho w \\
\rho E
\end{array}\right] \quad \mathbf{F}=\left[\begin{array}{c}
\rho u \\
\rho u u+p \\
\rho v u \\
\rho w u \\
u(\rho E+p)
\end{array}\right] \quad \mathbf{G}=\left[\begin{array}{c}
\rho v \\
\rho u v \\
\rho v v+p \\
\rho w v \\
v(\rho E+p)
\end{array}\right] \quad \mathbf{H}=\left[\begin{array}{c}
\rho w \\
\rho u w \\
\rho v w \\
\rho w w+p \\
w(\rho E+p)
\end{array}\right]
$$

$\mathbf{R}$ is the source terms due to the motion of the aircraft in the following form

$$
\mathbf{R}=\left[R_{\rho}, R_{V x}, R_{V y}, R_{V z}, R_{E}\right]^{T}
$$

where

$$
\begin{aligned}
& R_{\rho}=0 \\
& R_{E}=-\rho \mathbf{V}_{r} \cdot\left(\mathbf{a}_{0}+\dot{\omega} \times \mathbf{r}+\omega \times(\omega \times \mathbf{r})\right)
\end{aligned}
$$

and $R_{V x}, R_{V y}$ and $R_{V z}$ are the components of

$$
\mathbf{R}_{V}=-\rho\left(\mathbf{a}_{0}+\dot{\omega} \times \mathbf{r}+\omega \times(\omega \times \mathbf{r})+2 \omega \times \mathbf{V}_{r}\right) .
$$

In these equations and definitions, $\rho$ is the density, $p$ is the pressure, $E$ is the energy, $u, v$ and $w$ are the velocity components of $\mathbf{V}_{n}, \mathbf{a}_{0}$ is the acceleration vector of the origin of the moving frame, and $p, q$ and $r$ are the components of $\omega$, which is the angular velocity of the moving frame. 
Here, the unsteady sensitivity equations for computing the stability derivatives are briefly reviewed. According to Von Karman and Burgers [19], the unsteady aerodynamic forces and moments depend on the time histories of the motion of the aircraft. In longitudinal motions, this relationship in terms of pitching moment is

$$
C_{m}(t)=C_{m}(\alpha(\tau), q(\tau), V(\tau)) \quad \tau \in(-\infty, t] .
$$

If in Eq.(2) the moment coefficient depends on a short period of the past history [23], it is sufficient to assume that

$$
C_{m}(t)=C_{m}(\alpha(t), \dot{\alpha}(t), \ddot{\alpha}(t), \ldots, q(t), \dot{q}(t), \ddot{q}(t), \ldots, V(t), \dot{V}(t), \ddot{V}(t), \ldots) .
$$

In [21], besides Eq.(3), it is further assumed that at a fixed point in the non-inertial frame of reference, the conservative variables of the flow field can be also expressed by

$$
\mathbf{U}(t, x, y)=\mathbf{U}(\alpha(t), \dot{\alpha}(t), \ddot{\alpha}(t), \ldots, q(t), \dot{q}(t), \ddot{q}(t), \ldots, V(t), \dot{V}(t), \ddot{V}(t), \ldots ; x, y) .
$$

Eq. (4) is sufficient for deriving the unsteady sensitivity equations [21]

$$
\begin{aligned}
& \frac{\partial}{\partial t}\left(\mathbf{U}_{\gamma}\right)+\frac{\partial}{\partial x}\left(\mathbf{F}_{\gamma}\right)+\frac{\partial}{\partial y}\left(\mathbf{G}_{\gamma}\right)+\frac{\partial}{\partial z}\left(\mathbf{H}_{\gamma}\right)=\mathbf{R}_{\gamma} \\
& \frac{\partial}{\partial t}\left(\mathbf{U}_{\dot{\gamma}}\right)+\frac{\partial}{\partial x}\left(\mathbf{F}_{\dot{\gamma}}\right)+\frac{\partial}{\partial y}\left(\mathbf{G}_{\dot{\gamma}}\right)+\frac{\partial}{\partial z}\left(\mathbf{H}_{\dot{\gamma}}\right)=\mathbf{R}_{\dot{\gamma}}-\frac{\partial \mathbf{U}}{\partial y} \\
& \frac{\partial}{\partial t}\left(\mathbf{U}_{\ddot{\gamma}}\right)+\frac{\partial}{\partial x}\left(\mathbf{F}_{\ddot{\gamma}}\right)+\frac{\partial}{\partial y}\left(\mathbf{G}_{\ddot{\gamma}}\right)+\frac{\partial}{\partial z}\left(\mathbf{H}_{\ddot{\gamma}}\right)=\mathbf{R}_{\ddot{\gamma}}-\frac{\partial \mathbf{U}}{\partial \dot{\gamma}}
\end{aligned}
$$

where $\gamma$ is any one of $\alpha, q$ and $V$, and $\mathbf{U}_{\gamma}, \mathbf{U}_{\dot{\gamma}}$ and $\mathbf{U}_{\ddot{\gamma}}$ are called the sensitivity derivatives. Eq.(5) can be solved together with Eq.(1) to predict the sensitivity derivatives.

The sensitivity equations are passive equations depending on the solution of the flow governing equations. In Eq.(5), it is shown that the sensitivity derivatives with respect to $\dot{\gamma}$ and $\ddot{\gamma}$ depend on those with respect to $\gamma$ and $\dot{\gamma}$ respectively. Therefore, in practice, we firstly solve the flow governing equations, secondly solve the sensitivity equations with respect to $\gamma$ to predict the static derivatives, and then solve the sensitivity equations with respect to $\dot{\gamma}$ (and higher order terms when necessary) to compute the dynamic derivatives. The sequence of solution procedures for predicting the longitudinal stability derivatives is shown in Fig. 1. The computational cost of the present methods is closely related to the number of sensitivity equations being solved. As shown in Fig. 1, if the sensitivity equations with respect to $\alpha, q, V$ and $\dot{\alpha}, \dot{q}, \dot{V}$ are solved, the total equations to be solved will be 7 times as many as the flow governing equations. Therefore, the computational effort of the present approach is very large in general. In the present paper, only the sensitivity equations corresponding to the inviscid Euler equations are solved to save the computational cost.

The sensitivity equations and the flow governing equations are in a similar form and can be solved using basically the same numerical schemes. In the present paper, a finite volume solver in terms of the multi-block structured grids is used to solve both Eqs. (1) and (5). A reconstruction procedure based on the minimized dispersion and controllable dissipation $[24,25]$ is employed to compute the left and right states of conservative variables at the cell interface. The HLL Riemann solver [26] is used to compute the numerical flux of both the flow governing equations and the sensitivity equations. The dual time stepping LU-SGS technique [27] is used for temporal discretization. The 


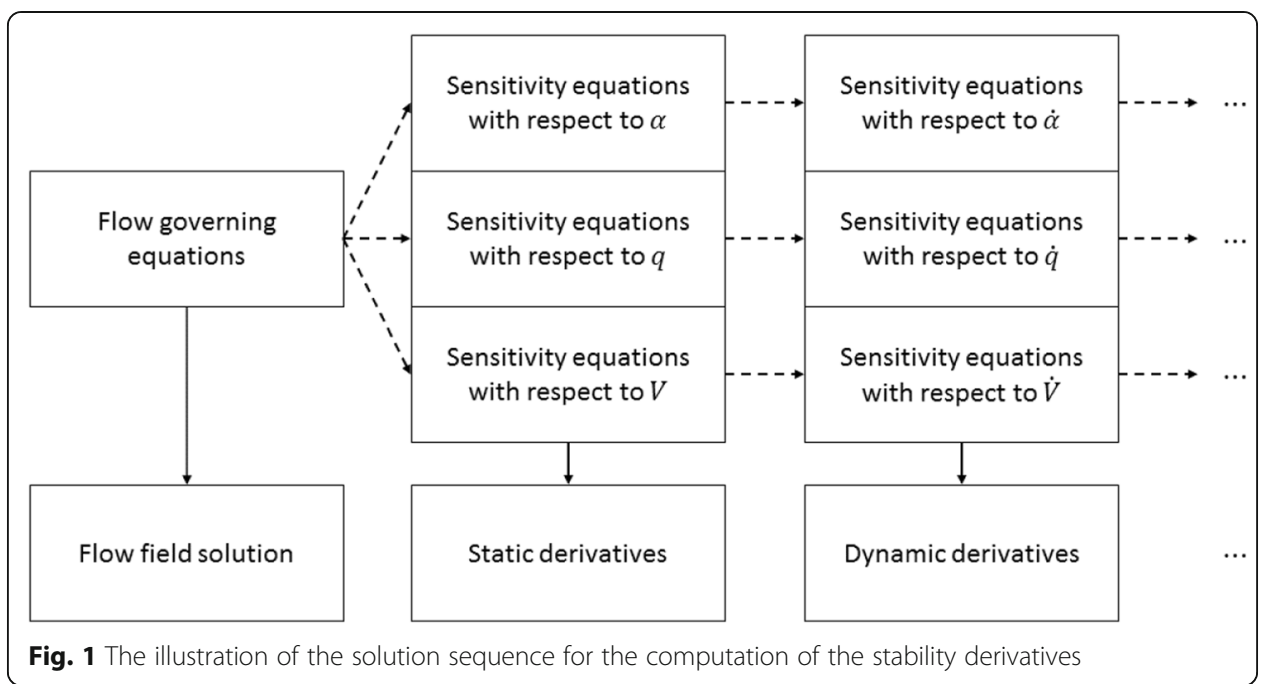

validation test cases for this numerical procedure can be found in [25]. The boundary conditions of the sensitivity equations can be straightforwardly derived from the boundary conditions for the flow governing equations. For example, the boundary conditions for the inviscid wall is

$$
\mathbf{V}_{r} \cdot \mathbf{n}=0
$$

in the non-inertial frame of reference. The corresponding wall boundary conditions for the sensitivity equations with respect to $\gamma$ and $\dot{\gamma}$ are respectively

$$
\left(\mathbf{V}_{r}\right)_{\gamma} \cdot \mathbf{n}=0
$$

and

$$
\left(\mathbf{V}_{r}\right)_{\dot{\gamma}} \cdot \mathbf{n}=0 .
$$

The far-field boundary conditions are handled using characteristic approaches based on the Riemann invariants in the boundary normal directions. The Riemann invariants can be also differentiated with respect to $\gamma$ and $\dot{\gamma}$ to obtain the boundary conditions for the corresponding sensitivity equations.

The predicted sensitivity derivatives can be used to compute the stability derivatives with respect to $\gamma, \dot{\gamma}, \cdots$ directly. For example, knowing the definition of the moment coefficient

$$
C_{m}=\left[\oiint_{\Omega} \mathbf{r} \times p \mathbf{n} d s\right] /\left[\frac{1}{2} \rho V_{\infty}^{2} S L\right],
$$

the stability derivative $C_{m y}$ is computed by

$$
\left(C_{m}\right)_{\gamma}=\left[\oiint_{\Omega} \mathbf{r} \times p_{\gamma} \mathbf{n} d s\right] /\left[\frac{1}{2} \rho V_{\infty}^{2} S L\right],
$$

where the sensitivity derivative $p_{\gamma}$ can be deduced from $\mathbf{U}_{\gamma} .\left(C_{m}\right)_{\dot{\gamma}}$ can be computed in a similar way.

The present method for computing the stability derivatives by solving the sensitivity equations is a entirely new approach. Although its theory has been presented in [21], 
some features of this approach are not studied in detail. Therefore, it is necessary to discuss further on these features especially for three-dimensional problems.

The first one is the aerodynamic model. In the present approach, we only need to know the abstract relation between the aerodynamic force/moment and the motion variables that is shown in Eq.(3). Most of other CFD based approach requires the prescription of an explicit aerodynamic model. For example, in [14], a steady reference motion is firstly given, and the increment of the aerodynamic force/moment is assumed to be the linear function of the increments of the state variables of a perturbative motion. On the other hand, the present approach does not require a steady reference motion. And the stability derivatives can be computed for any maneuver motion of the aircraft.

The second one is the dependency of the stability derivatives on the motion variables. In traditional approach for computing the pitching stability derivatives [14], the stability derivatives are only related to the steady reference motion and the reduced frequency. As a result, the static stability derivatives are assumed to be constant. However, in the present approach, it is easy to derive from Eq.(3) that

$$
\left(C_{m}\right)_{\alpha}=\frac{\partial C_{m}}{\partial \alpha}(\alpha(t), \dot{\alpha}(t), \ddot{\alpha}(t), \ldots, q(t), \dot{q}(t), \ddot{q}(t), \ldots, V(t), \dot{V}(t), \ddot{V}(t), \ldots) .
$$

Therefore, for a general maneuver motion of the aircraft, the static derivative $\left(C_{m}\right)_{\alpha}$ is also time-varying. This relation reveals that the stability derivatives are not only affected by the reference motion, but also by the perturbative motion. For other stability derivatives including the dynamic stability derivatives, the same conclusion can be also drawn.

The third one is that the present approach is capable of computing all of the static and dynamic derivatives in a single maneuver motion as long as the corresponding sensitivity equations are solved. In the case of the forced sinusoidal motion around the aircraft's center of gravity, instead of computing $C_{m_{\dot{\alpha}}}+C_{m_{q}}, C_{m_{\dot{\alpha}}}$ and $C_{m_{q}}$ can be computed individually. According to Eq.(7), they are computed respectively by

$$
\left(C_{m}\right)_{\dot{\alpha}}=\left[\oiint_{\Omega} \mathbf{r} \times p_{\dot{\alpha}} \mathbf{n} d s\right] /\left[\frac{1}{2} \rho V_{\infty}^{2} S L\right]
$$

and

$$
\left(C_{m}\right)_{q}=\left[\oiint_{\Omega} \mathbf{r} \times p_{q} \mathbf{n} d s\right] /\left[\frac{1}{2} \rho V_{\infty}^{2} S L\right] .
$$

In these formulations, $p_{\dot{\alpha}}$ and $p_{q}$ are computed by the solutions of Eq.(5). The present method gives the time histories of $C_{m_{\dot{\alpha}}}$ and $C_{m_{q}}$ so that their dependency on the reduced frequency can be shown. For traditional methods, the pitching oscillations can be used only to compute $C_{m_{\dot{\alpha}}}+C_{m_{q}}$. In order to compute both $C_{m_{\dot{\alpha}}}$ and $C_{m_{q}}$, besides the pitching oscillation, additional plunging oscillation should also be considered [28]. This approach is feasible only when the assumption is valid that the stability derivatives are solely related to the steady reference motion. However, according to the analysis presented above in the second feature of the present method, the stability derivatives are not only affected by the reference motion, but also by the perturbative motion. Therefore, using two different perturbative motions to separate $C_{m_{\dot{\alpha}}}$ and $C_{m_{q}}$ from $C_{m_{\dot{\alpha}}}+C_{m_{q}}$ will introduce additional errors. It is reported in [29] that the approach 


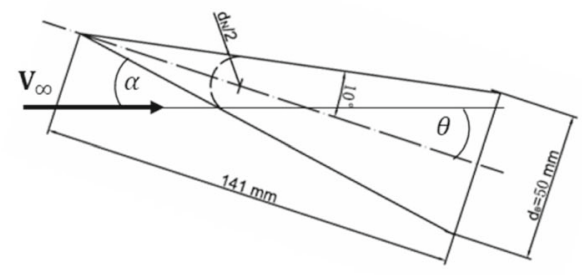

a The configuration of the cone, $\mathrm{d}_{\mathrm{N}} / \mathrm{d}_{\mathrm{B}}=0.4$

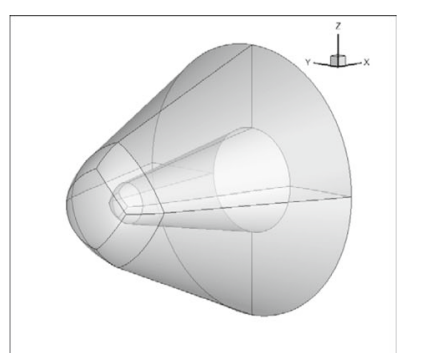

b The computational domain of the blunt cone without bottom region

Fig. 2 The geometry and the computational domain of the blunt cone. a The configuration of the cone, $d_{N} / d_{B}=0.4$. $\mathbf{b}$ The computational domain of the blunt cone without bottom region

using pitching and plunging oscillations to compute $C_{m_{\dot{\alpha}}}$ and $C_{m_{q}}$ showed great frequency dependency. To reduce this dependency, it is proposed in [29] that $C_{m_{\dot{\alpha}}}$ and $C_{m_{q}}$ are computed using the looping and heaving motions. In the present approach, any single maneuver motion can be used to $C_{m_{\dot{\alpha}}}$ and $C_{m_{q}}$ directly, which totally removes the ambiguity in computing these stability derivatives.

The last feature is that the present method gives not only the stability derivatives directly by solving the sensitivity equations, but also the distribution of the sensitivity derivatives such as $p_{\gamma}$ and $p_{\dot{\gamma}}$. The distribution of the sensitivity derivatives such as $p_{\gamma}$ and $p_{\dot{\gamma}}$ appeared in Eq. (7) gives additional information which is not available for the traditional methods. In the present paper, we propose to use this information to evaluate the local contribution of a particular element of an aircraft to the stability derivatives of the whole aircraft. To this end, the surface of an aircraft is divided in to $N$ parts with

$$
\Omega=\sum_{i}^{N} \Omega_{i}
$$

On each part, the contribution to the moment derivatives can be computed by

$$
\left(m_{y}\right)_{\gamma, i}=\oiint_{\Omega_{i}} \mathbf{r} \times p_{\gamma} \mathbf{n} d s .
$$

The moment stability derivative of the aircraft $\left(m_{y}\right)_{y}$ is computed as

$$
\left(m_{y}\right)_{\gamma}=\sum_{i}^{N}\left(m_{y}\right)_{\gamma, i}
$$

which is nondimensionalized to obtain $C_{m_{\gamma}}$. The term $\left(m_{y}\right)_{\gamma, i}$ is called the Moment of Pressure Derivatives (MPD) in this paper. This value indicates the amount of contribution of any particular body surface to the overall stability derivatives. The importance of MPD is to identify the crucial locations that affect the stability of an aircraft so that local measurement can be introduced to effectively stabilize or destabilize the aircraft. In conventional methods based on the CFD technique, since $p_{\gamma}$ is not known, it is impossible to calculate the local contribution to the stability derivatives although it is possible to calculate the local contribution to the moment coefficient. 


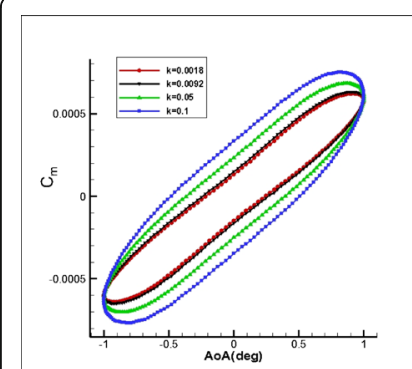

a $C_{m}$

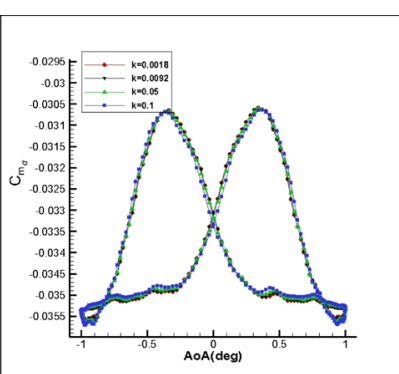

b $\quad C_{m_{\alpha}}$

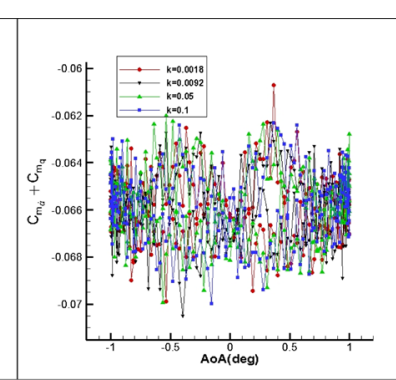

c $\quad C_{m_{\dot{\alpha}}}+C_{m_{q}}$

Fig. $3 C_{m}$ and its derivatives at $a_{0}=0^{\circ}, X_{c g} / L=0.70 . \mathbf{a} C_{m} \cdot \mathbf{b} C_{m_{a}}$. c $C_{m_{\dot{a}}}+C_{m_{q}}$

\section{Results and discussions}

\subsection{The test case}

The stability derivatives of blunt cones undergoing the forced oscillations was studied using the wind-tunnel experiments in [30,31]. The configuration and geometry parameters of the cones are shown in Fig. 2a. The ratio between the nose diameter and the base diameter $d_{N} / d_{B}$ is 0.4 .

In this section, we will study this test case numerically. For the forced pitching oscillation around a fixed point, the pitch angle equals to the angle of attack

$$
\alpha=\theta,
$$

and the forced oscillation is in the following form [32]

$$
\alpha=\theta=\alpha_{0}+\alpha_{1} \sin \left(\frac{2 V_{\infty} k t}{L}\right)
$$

where $\alpha_{0}$ and $\alpha_{1}$ are the mean value and amplitude of the oscillation, $L$ is the characteristic length of the cone, $V_{\infty}$ is the velocity of the freestream, and $k$ is the reduced frequency. The model was tested according to different rotating centers $\left(X_{c g}=0.70 L\right.$ and $\left.X_{c g}=0.75 L\right)$. The two sets of experimental conditions are named as Xcg70 and Xcg75 in this paper. The computational domain is shown in Fig. 2b with about 1.6 million cells.

The stability derivatives of the moment coefficient with respect to $\alpha, \dot{\alpha}$ and $q(q=\dot{\theta})$ are presented in this paper. It should be noted that the stability derivatives under any

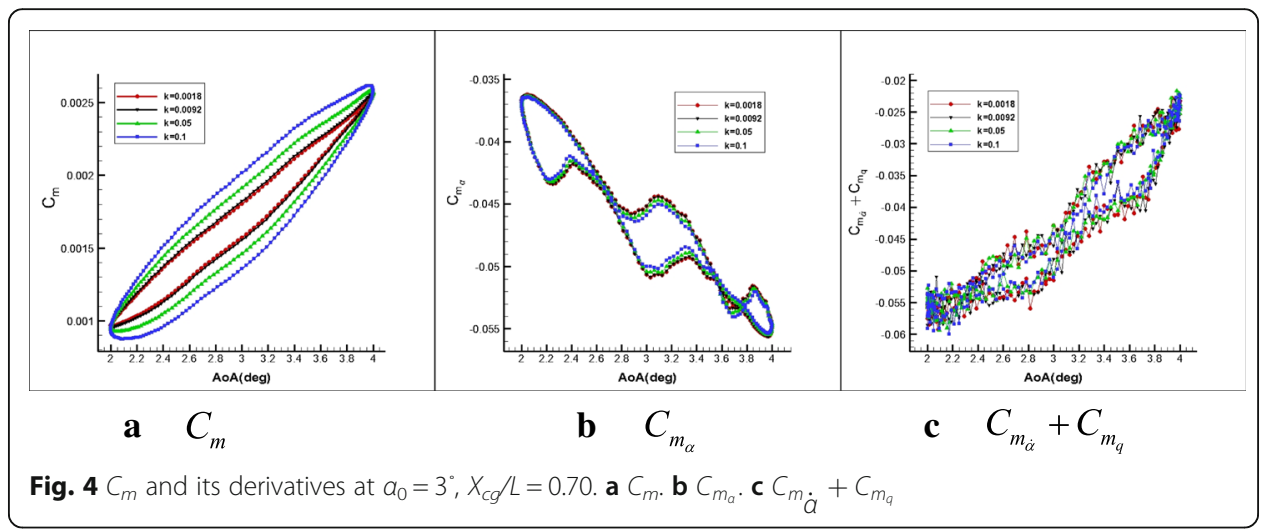




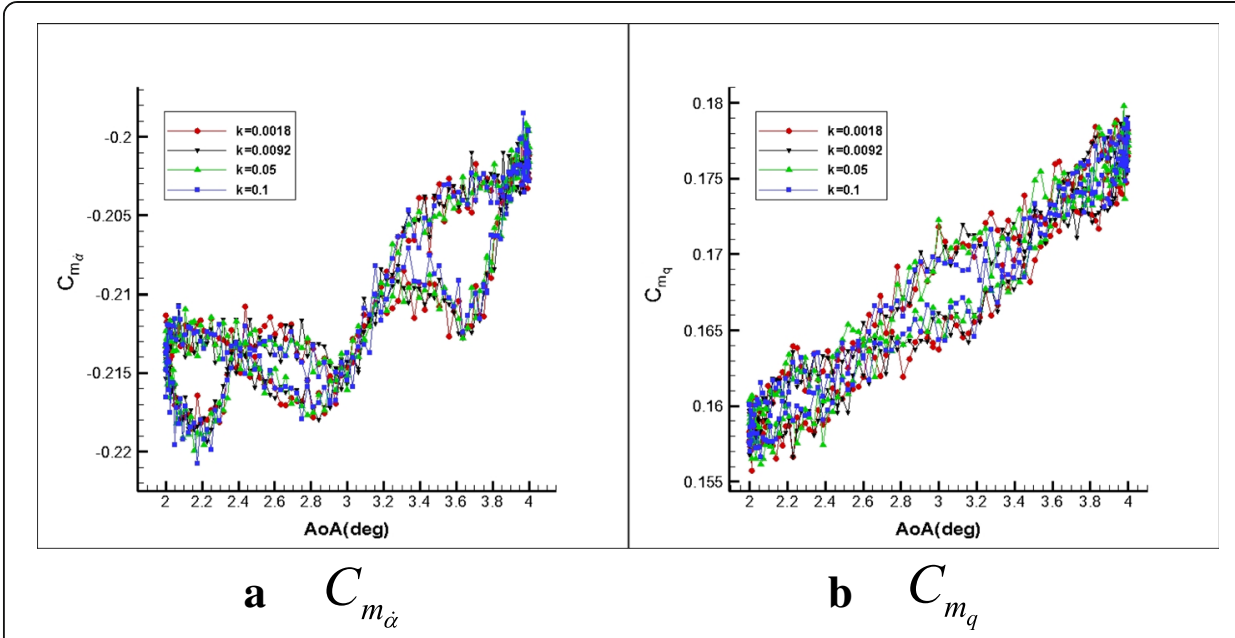

Fig. 5 The components of the dynamic derivatives at $a_{0}=3^{\circ}$ for $X_{c g} L=0.70 . \mathbf{a} C_{m_{\dot{a}}} \cdot \mathbf{b} C_{m_{q}}$

maneuver can be computed, while in this paper only the derivatives of the pitch damping motion are computed.

\subsection{The stability derivatives}

The results of Xcg70 are discussed here to show the stability derivatives during the pitch damping motions. The amplitude of the angle of attack is $1^{\circ}$, and the Mach number is 6.85. The range of reduced frequency $k$ of the experiments is from 0.0018 to 0.0092. In order to observe and analyze the influences of the pitching frequency, more frequencies are used in this test case.

Figure 3 and Fig. 4 show the variation of the pitching moment coefficient and its derivatives versus the angle of attack, where $\alpha_{0}=0^{\circ}$ and $3^{\circ}$. It can be observed that the stability derivatives are not constant, and they are changing with the angle of attack. This is in contradiction with some linear aerodynamic models in which the stability derivatives are functions of the mean angle of attack only.

It is also clear that there is the time-lagged effect in both the momentum coefficient and its stability derivatives. Further study reveals an interesting phenomenon. The hysteresis effects of the moment coefficients become stronger as the reduced frequency

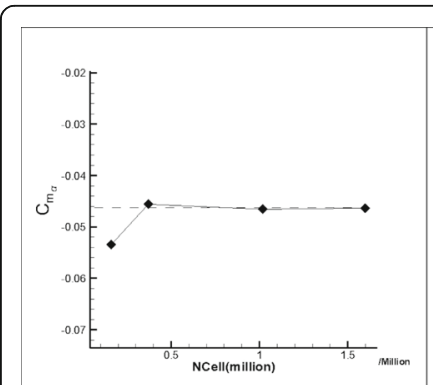

a $\quad C_{m_{\alpha}}$

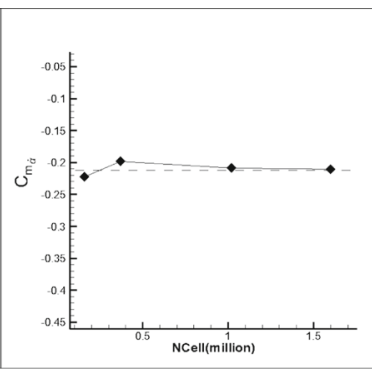

b $C_{m_{\dot{\alpha}}}$

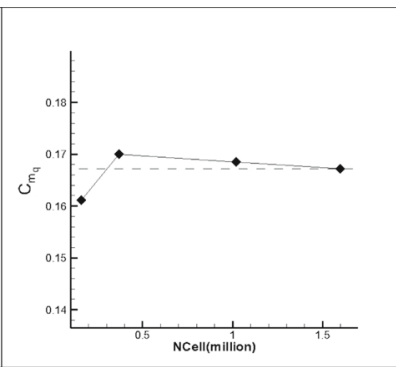

c $C_{m_{q}}$

Fig. 6 The time-average values of $C_{m_{a}}, C_{m_{\dot{a}}}$ and $C_{m_{q}}$ versus different numbers of cells. a $C_{m_{a}}$. b $C_{m_{\dot{a}}} \cdot \mathbf{c} C_{m_{q}}$ 
increases. However, the hysteresis effects of the stability derivatives are not very sensitive to the reduced frequency. Another feature of the stability derivatives is that the static derivative $C_{m_{\alpha}}$ is smoothly changed with the angle of attack, while there are oscillations in the dynamic damping derivative $C_{m_{\dot{\alpha}}}+C_{m_{q}}$. This result indicates that the higher order sensitivity derivatives are more sensitive to the flow field prediction.

A distinctive feature of the present method is that the dynamic stability derivatives $C_{m_{\dot{\alpha}}} \dot{\alpha}$ and $C_{m_{q}}$ can be predicted separately. The results of $C_{m_{\dot{\alpha}}}$ and $C_{m_{q}}$ are shown in Fig. 5. It is found that the $C_{m_{\dot{\alpha}}}$ is negative and thus stabilizes the motion, and on the other hand the $C_{m_{q}}$ is positive and tends to make the motion unstable. Using conventional methods, only $C_{m_{\dot{\alpha}}}+C_{m_{q}}$ can be predicted, and as the result, the destabilization effect of $C_{m_{q}}$ cannot be revealed.

\subsection{Grid convergence}

The grid convergence is important to ensure that the numerical solutions of the stability derivatives are accurate on given grids. In this subsection, the grid convergence of the numerical methods for solving the sensitivity equations is verified by increasing the number of grids gradually from 0.17 million to 1.60 million. The mean angle of attack $\alpha_{0}$ is set to $3^{\circ}$, and the amplitude of oscillation $\alpha_{1}$ is $1^{\circ}$. The position of the rotating center $X_{c g}=0.70 \mathrm{~L}$.

Figure 6 shows the results of the mean value of moment coefficient derivatives with respect to $\alpha, \dot{\alpha}$ and $q$ under different grid numbers. When the grid is refined, the tendency of convergence is observed in the numerical solutions. The differences for $C_{m_{\alpha}}, C_{m_{\dot{\alpha}}}$ and $C_{m_{q}}$ are $0.47 \%, 0.58 \%$ and $0.77 \%$ between the two most refined grids with $1.02 \mathrm{M}$ and $1.6 \mathrm{M}$ grids. In what follows, only the numerical results on the finest grid are shown.

\subsection{The comparison with experimental data}

In the experimental study [30,31], only the mean values of the stability derivatives are measured. In the present study, the mean values of the stability derivatives are

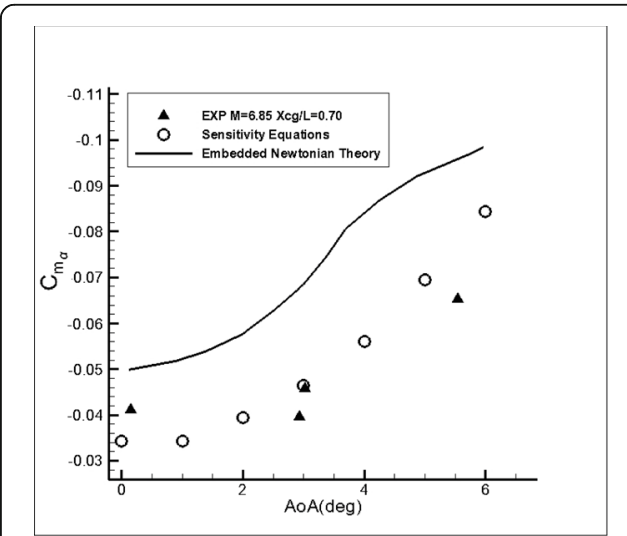

a Static derivatives of $X_{c g} / L=\mathbf{0 . 7 0}$

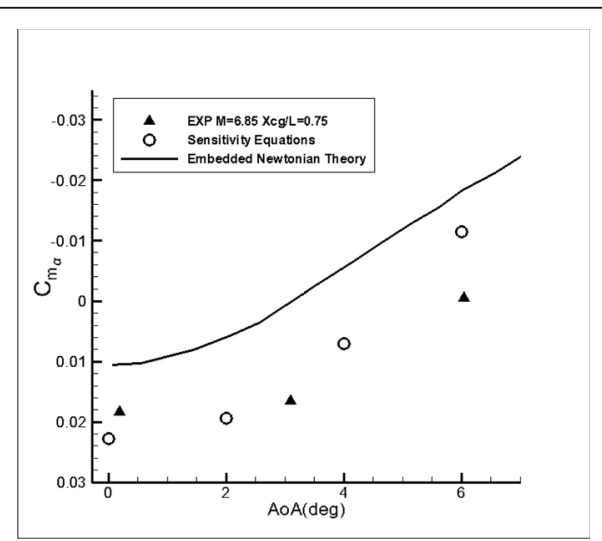

b Static derivatives of $X_{c g} / L=\mathbf{0 . 7 5}$

Fig. 7 Comparison of the static of theoretical, experimental and numerical methods for $X_{c g} / L=0.70$ and $X_{c g} / L=0.75$. a Static derivatives of $X_{c g} / L=0.70$. b Static derivatives of $X_{c g} / L=0.75$ 


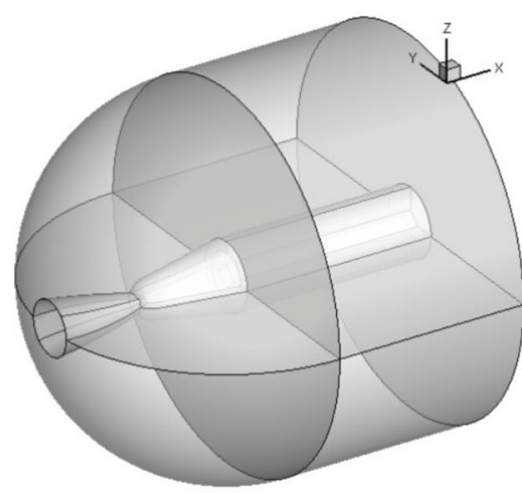

a Topology of blocks

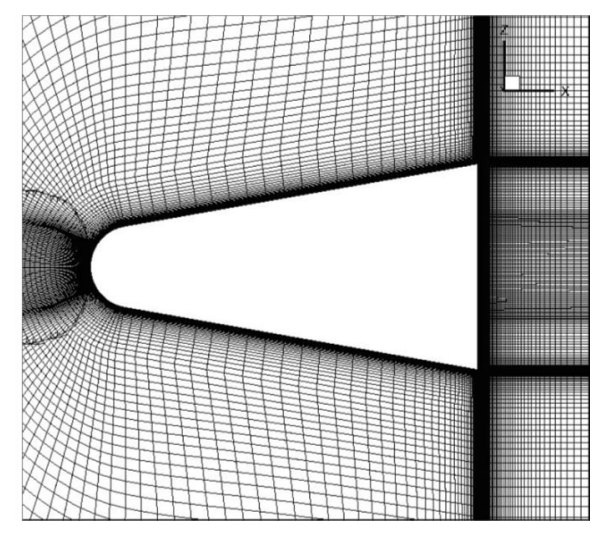

b Near view of the grid

Fig. 8 The refined grid considering the bottom regions. a Topology of blocks. $\mathbf{b}$ Near view of the grid

computed by the time-average of the instantaneous solutions of the stability derivatives on one period of the forced oscillation. Figure 7 shows the results of mean static derivatives at different mean angles of attack $\left(\leq 6^{\circ}\right)$ for both Xcg70 and Xcg75. The results are in reasonable agreement with the experimental data and are more accurate than the theoretical results of the embedded Newtonian theory [30, 31].

When the mean angle of attack increases further, the errors between the numerical and experimental results will become larger (not shown here). To explain this phenomenon, we notice that the computational domain which is shown in Fig. 2 does not include the bottom region of the blunt cone. When the angle of attack is large enough, the asymmetry of the flow field may have a large influence on the stability derivatives. Therefore, this test case is recomputed using the computational domain and corresponding grids shown in Fig. 8.

After considering the bottom effect, the static derivatives of Xcg75 at $\alpha_{0} \in\left[4^{\circ}, 10^{\circ}\right]$ are shown in Fig. 9. In this results, the predicted static stability derivatives are in good agreement with the experimental results. When the angle of attack increases to an even large value, the inviscid nature of Euler equations may prevent an accurate prediction of the stability derivatives since the flow separations at the bottom region are dominated by the viscous effect. The use of the Navier-Stokes equations for predicting the sensitivity derivatives is very expensive, which will be studied in the future work.

Figure 10 shows the mean dynamic derivatives $C_{m_{\dot{\alpha}}}+C_{m_{q}}$ after considering the bottom effect. It is shown that when the angle of attack is smaller than $10^{\circ}$, the agreement between the numerical results and the experimental results are reasonable although the errors are considerably larger than the static derivatives. When the angle of attack is larger than $10^{\circ}$, the errors become even larger. This is also an indication that the Euler equations may not be appropriate when the angle of attack is large. However, the tendency of the dynamic stability derivatives with respect to the angle of attack is better predicted by the present method when compared with the embedded Newtonian theory.

We note further that in the experiment, only $C_{m_{\dot{\alpha}}}+C_{m_{q}}$ can be predicted directly. On the other hand, the present method can compute $C_{m} \dot{\alpha}$ and $C_{m_{q}}$ separately. The mean values of these derivatives are shown in Fig. 11. It is found that $C_{m_{\dot{\alpha}}}$ and $C_{m_{q}}$ are usually of opposite signs and tend to eliminate each other, which makes the variation of 


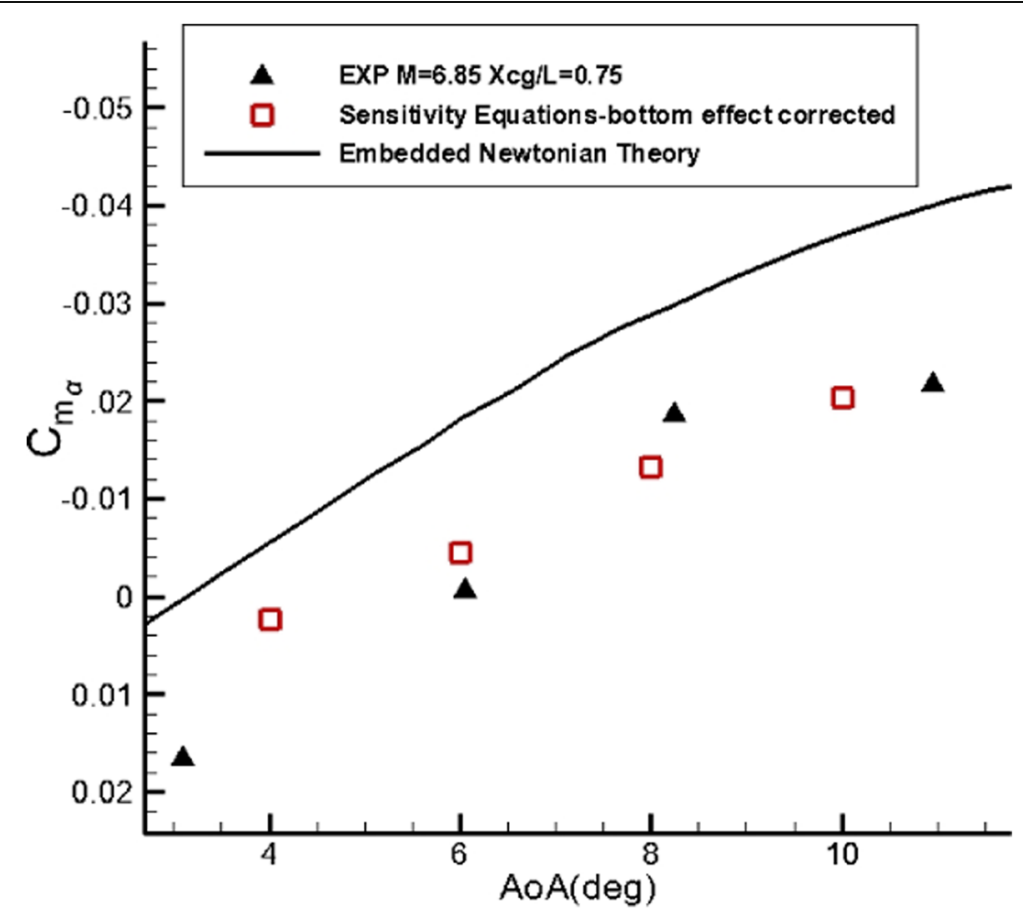

Fig. 9 Comparison of the static moment derivative between theoretical, experimental and numerical results after considering the bottom effect

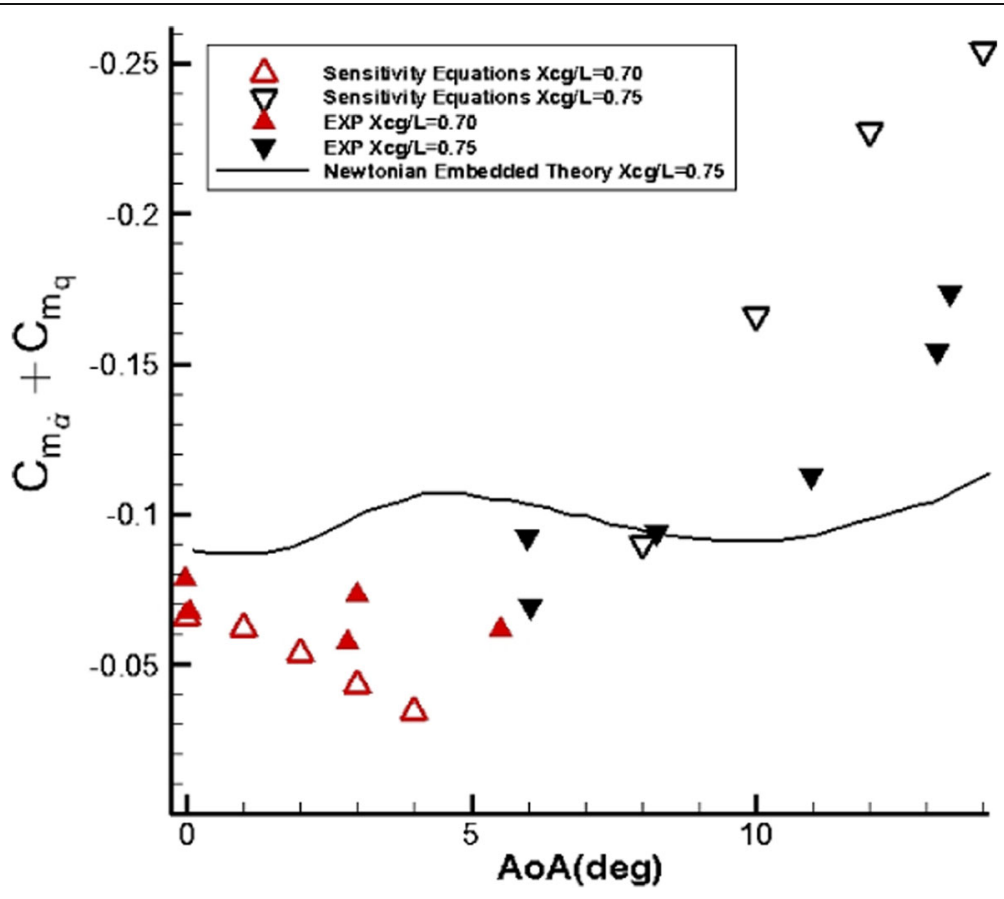

Fig. 10 Comparison of the dynamic moment derivatives between the theoretical, experimental and numerical results after considering the bottom effect 


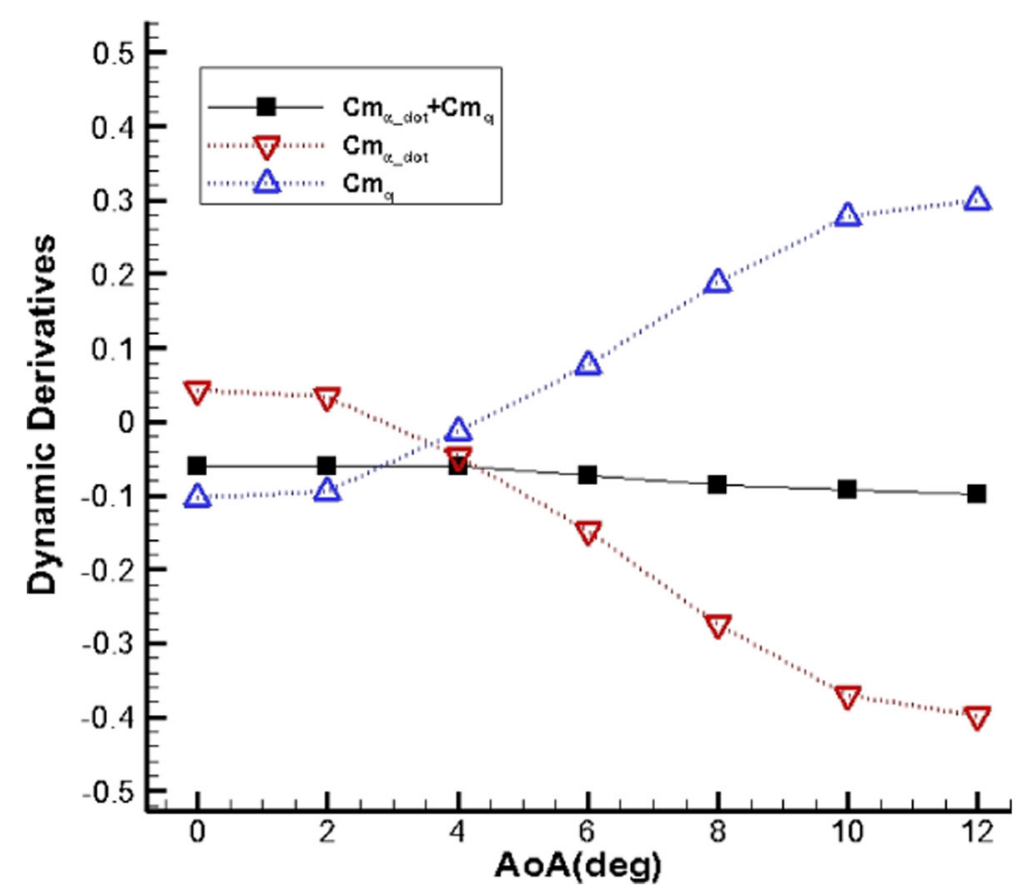

Fig. 11 Components of Dynamic derivatives

$C_{m_{\dot{\alpha}}}+C_{m_{q}}$ being much smaller than its individual components. This phenomenon shows the importance of predicting $C_{m_{\dot{\alpha}}}$ and $C_{m_{q}}$ separately.

\subsection{Evaluation of the local contribution of the blunt cone to the stability derivatives using the sensitivity derivatives}

The MPD defined in Eq.(8) is used to evaluate the contribution of local surfaces of the blunt cone to the moment stability derivatives. For the present case, the surface of the cone is divided into three parts, namely the head, body and bottom parts which are shown in Fig. 12. Their contributions to the stability derivatives are given in Table 1 when the mean angles of attack is $8^{\circ}$. In Table 1 , the positive percentage means the stabilization effect while the negative percentage means destabilization effect. For static stability derivative $C_{m_{\alpha}}$, the stabilization effect is achieved by the head of the blunt cone, and the body and bottom parts both destabilize the cone. And the head region plays the most important role in the static stability. For the dynamic stability derivative $C_{m_{\dot{\alpha}}}+C_{m_{q}}$, all three parts have the stabilization effect, and the body part provides about $80 \%$ of the overall stability. The bottom effect can be also quantitatively identified using the MPD. It can be deduced from Table 1 that in terms of the absolute value of MPD, the bottom region contributes $6.4 \%$ of the total static stability, and contributes $4.9 \%$ of the total dynamic stability.

\section{Conclusion}

In this paper, the numerical method for evaluating the stability derivatives based on the unsteady sensitivity equations is extended to 3D cases. This method takes the unsteady effects into consideration and can be used to predict any stability derivative by solving 


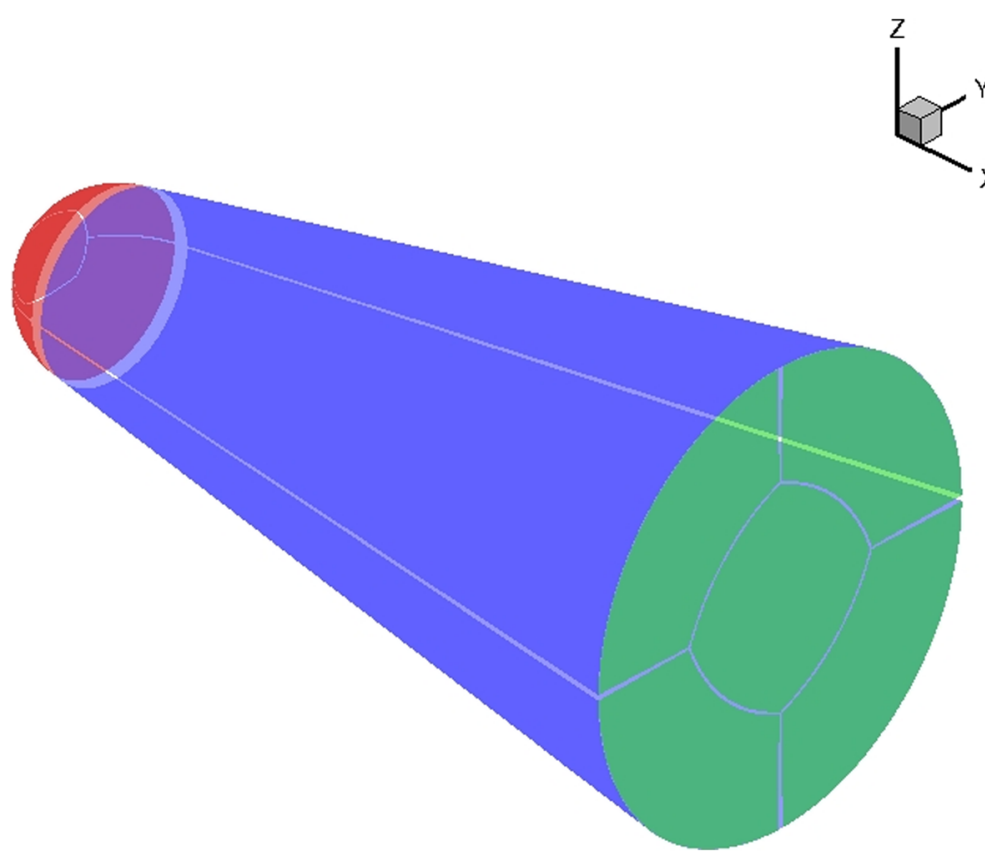

Fig. 12 The sketch diagram of components of the blunt cone, red-head, blue-body, green-bottom

the unsteady flow and the corresponding sensitivity equations. There are two remarkable features of this method. One of them is the possibility to distinguish the components of dynamic derivatives caused by different state parameters. The other feature is that the MPD can be used to predict the contribution of each part of the aircraft to the overall stability quantitatively. The supersonic blunt cone is tested to validate this method.

For the stability derivatives of the blunt cone in hypersonic flow with Mach number 6.85 , the numerical results show that when the angle of attack is not very large, both static and dynamic stability derivatives can be predicted in reasonable accuracy which is usually higher than the embedded Newtonian theory. For the static stability, the stabilization effect is achieved by the head of the blunt cone, and the body and bottom parts both destabilize the cone. For the dynamic stability, $C_{m_{\dot{\alpha}}}$ and $C_{m_{q}}$ are usually of opposite signs and tend to eliminate each other, which makes the variation of $C_{m_{\dot{\alpha}}}+C_{m_{q}}$ being much smaller than its individual components. The body part provides about $80 \%$ of the overall dynamic stability.

\section{Nomenclature}

$\alpha$ angle of attack, shown in Fig. 2a

Table 1 Contribution of head, body and bottom part to the overall stability

\begin{tabular}{lllll}
\hline Components & Static derivatives & Contribution \% & Dynamic derivatives & Contribution \% \\
\hline Head & $-6.976 \mathrm{E}-01$ & $728.77 \%$ & $-1.338 \mathrm{E}-02$ & $14.88 \%$ \\
Body & $5.187 \mathrm{E}-01$ & $-541.89 \%$ & $-7.215 \mathrm{E}-02$ & $80.26 \%$ \\
Bottom & $8.315 \mathrm{E}-02$ & $-86.88 \%$ & $-4.370 \mathrm{E}-03$ & $4.86 \%$ \\
Sum & $-9.572 \mathrm{E}-02$ & $100 \%$ & $-8.990 \mathrm{E}-02$ & $100 \%$ \\
\hline
\end{tabular}


$\theta$ pitching angle, shown in Fig. 2a

$q$ pitching angular velocity

$V$ magnitude of velocity

$\gamma$ any one of $\alpha, q$ and $V$

$C_{m}$ moment coefficient, defined in Eq.(6)

$\left(C_{m}\right)_{\gamma}$ Stability derivatives of $C_{m}$ with respected to $\gamma$

\section{Acknowledgements}

The authors would like to thank the national numerical wind tunnel project and the national key research and development program of China for their financial support.

\section{Authors' contributions}

The methods are developed by both authors based on the idea of the corresponding author, and the coding and numerical simulations are carried out by the first author. Both authors read and approved the final manuscript.

\section{Funding}

This work is supported by national numerical wind tunnel project under contract number 2018-ZT4A07 and 2016YFA0401200 of national key research and development program of China.

\section{Availability of data and materials}

Parts of the data and materials are available upon request.

\section{Competing interests}

The authors declare that they have no competing interests.

Received: 27 February 2019 Accepted: 10 June 2019

Published online: 20 July 2019

\section{References}

1. Chambers JR, Hall RM (2004) Historical review of uncommanded lateral-directional motions at transonic conditions. J Aircr 41(3):436-447

2. Hall RM, Biedron RT et al (2005) Computational methods for stability and control (COMSAC): the time has come, AIAA Atmospheric Flight mechanics conf. and exhibit, AIAA, p 6121

3. Bryan GH (1911) Stability in aviation. Macmillan, New York

4. Tobak M (1954) On the use of indicial function concept in the analysis of unsteady motions of wings and wing-tail combinations, NACA Report 1188

5. Tobak M, Schiff LB (1976) On the formulation of the aerodynamic characteristics in aircraft dynamics, NACA TR R-456

6. Goman MG, Zagainov GI (1997) Application of bifurcation methods to nonlinear flight dynamics problems. Prog Aerosp Sci 33(9-10):539-586

7. Klein V, Noderer KD (1994) Modeling of aircraft unsteady aerodynamic characteristics, NASA Technical Memorandum 109120

8. Greenwell D (2004) A review of unsteady aerodynamic modelling for flight dynamics of maneuverable aircraft, AIAA, p 5276

9. Ghoreyshi M, Jirasek A, Cummings RM (2014) Reduced order unsteady aerodynamic modeling for stability and control analysis using computational fluid dynamics. Prog Aerosp Sci 71:167-217

10. Ghoreyshi M, Cummings RM (2012) Unsteady aerodynamics modeling for aircraft maneuvers: a new approach using time-dependent surrogate modeling, 30th AIAA Applied Aeroedynamics Conference, AIAA, p 3327

11. Mader CA, Martins JA (2011) Computation of aircraft stability derivatives using an automatic differentiation Adjoint approach. AIAA J 49(12):2737-2750

12. Da Ronch A, Ghoreyshi M, Badcock KJ (2011) On the generation of flight dynamics aerodynamic tables by computational fluid mechanics. Prog Aerosp Sci 47(8):597-620

13. Klein V, Murphy PC, Curry TJ, Brandon J (1997) Analysis of wind tunnel longitudinal static and oscillatory data of the F $-16 \mathrm{XL}$ aircraft, NASA tm-97-206276

14. Da Ronch A, Vallespin D, Ghoreyshi D, Badcock K (2010) Computation of Dynamic Derivaitves Using CFD, 28th AIAA Applied Aerodynamic Conference, AIAA, p 4562

15. Da Ronch A, Ghoreyshi D, Badcock K, Görtz K, Widhalm S, Dwight M, Champobasso M (2010) Linear Frequency Domain and Harmonic Balance Predictions of Dynamic Derivatives, 28th AIAA Applied Aerodynamic Conf., AIAA, p 4699

16. Da Ronch A, Vallespin D, Ghoreyshi M, Badcock K (2012) Evaluation of dynamic derivatives using computational fluid dynamics. AIAA J 50(2):470-484

17. Görtz S, McDaniel DR, Morton SA (2007) Towards an efficient aircraft stability and control analysis capability using highfidelity CFD, AIAA, p 1053

18. Dean JP, Morton SA, McDaniel DR et al (2008) Aircraft stability and control characteristics determined by system identification of CFD simulations, AIAA, p 6378

19. Godfrey AG, Cliff R (1998) Direct calculation of aerodynamic force derivatives: a sensitivity-equation approach AIAA 98-0363

20. Limache A, Cliff E (2000) Aerodynamic sensitivity theory for rotary stability derivatives. J Aircr 37:676-683

21. Ren YX (2008) Evaluation of the stability derivatives using the sensitivity equations. AIAA J 46(4):912-917 
22. Lei GD, Ren YX (2011) Computation of the stability derivatives via CFD and the sensitivity equations. Acta Mech 27(2):179-188

23. Murman SM (2007) Reduced-frequency approach for calculating dynamic derivatives. AIAA J 45(6):1161-1168

24. Sun ZS, Ren YX (2011) A class of finite difference schemes with low dispersion and controllable dissipation for DNS of compressible turbulence. J Comput Phys 230:4616-4635

25. Wang QJ, Ren YX, Sun ZS, Sun YT (2013) Low dispersion finite volume scheme based on reconstruction with minimized dispersion and controllable dissipation. Sci China Phys Mech Astron 56(2):423-431

26. Harten A, Lax PD, Van Leer B (1983) On upstream differencing and Godunov-type schemes for hyperbolic conservation Laws. SIAM Rev 25(1):35-61

27. Jameson A, Turkel E (1981) Implicit scheme and LU-decompositions. Math Comput 37:385-397

28. Giesing JP, Rodden WP (1970) Application of oscillatory aerodynamic theory to estimation of dynamic stability derivatives. J Aircr 7(3):272-275

29. Gili P, Visone M, Lerro A (2015) A new approach for the estimation of longitudinal damping derivatives: CFD validation on NACA 0012. WSEAS Trans Fluid Mech 10:137-145

30. East RA, Qasrawi AMS, Khalid M (1978) An experimental study of the hypersonic dynamic stability of pitching blunt conical and hyper-ballistic shapes in a short running time facility, NATO AGARD CP-235

31. Hutt GR, East RA (1985) Optical techniques for model position measurement in dynamic wind tunnel testing. Meas Control 18:99-101

32. East RA, Hutt GR (1988) Comparison of predictions and experimental data for hypersonic pitching motion stability. J Spacecr 25(3):225-233

\section{Publisher's Note}

Springer Nature remains neutral with regard to jurisdictional claims in published maps and institutional affiliations.

Submit your manuscript to a SpringerOpen ${ }^{\circ}$ journal and benefit from:

- Convenient online submission

- Rigorous peer review

- Open access: articles freely available online

High visibility within the field

- Retaining the copyright to your article

Submit your next manuscript at $\boldsymbol{\nabla}$ springeropen.com 